\title{
Transfert de chaleur par métaux liquides
}

\section{Heat transfer by fluid metals}

\author{
PAR G. BRAUDEAU \\ CHEF DE LA DIVISION ÉTUDES GÉNÉRALES \\ AU SERYICE DES ÉTUdes ET REGHERChES hydRAULIQUES I'ÉLECTRICITĹ de FRANCE
}

\begin{abstract}
Les métaux liquides peuvent être utilisés comme fluides réfrigérants lorsque de grands flux de chaleur à des températures élevées imposent l'emploi d'un fluide chimiquement stable $\dot{a}$ faible tension de vapeur et $\dot{a}$ fort coefficient d'échange.

Sont plus particulièrement envisagés les métaux: alcalins, notamment le sodium et des alliages sodium-potassium, malgré une grande activité chimique qui nécessite l'élaboration d'une technologie spéciale.

Dans l'échange de chaleur en écoulement turbulent, la conduction moléculaire joute un rôle très important, et les théories classiques de la convection turbutente ne s'appliquent pas sans modifications,
\end{abstract}

\begin{abstract}
Liquid metals can be used as cooling fluids when large heat fuxes at high temperatures necessitate the use of a fluid which is chemically stable, has a low vapour pressure and a high heat trunsfer coefficient.

Those receibing special attention are metals of the alkali group, especially sodium and sodiumpotassium allols, despite their readiness to react chemically, which calls for special techniques to be developed.

Molecular conduction, playss a large role in the transfer of heat in turbulent flow conditions and the classical theories of turbulent convection cannot be applied withont modification.
\end{abstract}

\section{1. - GENERALITES}

L'eau est depuis toujours le fluide le plus largement utilisé pour extraire et transporter la chaleur.

La nécessité, dans certains cas, d'extraire des flux de chaleur importants à des températures élevées, disons de 400 à $800^{\circ}$ pour fixer les idćes, sans vaporisation de fluide, conduit à rechercher des corps moins volatils, glui puissent supporter de telles tempćratures en phase liquide sous des pressions raisonnables.

Les conditions mêmes d'emploi exigent de ces corps une parfaite stabilité chimique à chaud.

D'autre part, on doit leur demander d'avoir une chaleur spécifique suffisante pour favoriser l'échange et le transport des calories avec un débit, done une puissance de pomprage, aussi réduits que possible.

Ces diverses qualités sont réunies, à des degrés divers, par quelques métaux ou alliages, liquides aux températures que nous envisageons. Elles sont assorties toutefois de certains défauts qui n'en rendent pas toujours l'usage très facile.

Regardons seulement le tableau suivant, qui fournit les caractéristiques essentielles de quelques mélaux qui nous intéressent.

\begin{tabular}{|c|c|c|c|c|c|}
\hline & $N a$ & $\begin{array}{c}\text { Na K } \\
\text { Eutec- } \\
\text { lirque }\end{array}$ & 118 & $\mathrm{~Pb}$ & $\begin{array}{l}\text { Ph Bi } \\
\text { Eutce- } \\
\text { tigue }\end{array}$ \\
\hline Fusion $(" C) \ldots \ldots \ldots$ & 97,8 & -11 & $-38,9$ & 327,4 & 125 \\
\hline $\begin{array}{l}\text { Ebullition ("C) (pres- } \\
\text { sion atmospherique). }\end{array}$ & 883 & 784 & 357 & 1737 & 1676 \\
\hline Densité . . & $\left\{\begin{array}{l}0,854 \\
\left(400^{\prime \prime}\right)\end{array}\right.$ & {$\left[\begin{array}{c}0,775 \\
\left(400^{\circ}\right)\end{array} \mid\right.$} & $\left(\begin{array}{c}12,9 \\
\left(300^{\prime \prime}\right)\end{array}\right.$ & {$\left[\begin{array}{r}10,5 \\
(400 ")\end{array}\right]$} & 10,2 \\
\hline $\begin{array}{l}\text { Chaleur spécifique... } \\
\left(\mathrm{cal} / \mathrm{g}^{\circ} \mathrm{C}\right) \ldots \ldots\end{array}$ & $\left|\begin{array}{l}0,305: 5 \\
\left(400^{\circ}\right)\end{array}\right|$ & $\left(\begin{array}{l}0,210 \\
\left(.100^{\prime \prime}\right)\end{array}\right.$ & $(0,0325)$ & $\left(\begin{array}{c}0,037 \\
\left(4000^{\prime \prime}\right)\end{array}\right.$ & $\begin{array}{l}0,0355 \\
\left(4000^{\circ}\right)\end{array}$ \\
\hline $\begin{array}{l}\text { Conductivite thermique } \\
\quad\left(\mathrm{cal} / \mathrm{s} . \mathrm{cm} .{ }^{\circ} \mathrm{C}\right) \ldots \ldots\end{array}$ & $\left(\begin{array}{l}0,17 \\
\left(400^{\prime \prime}\right)\end{array}\right.$ & $\begin{array}{c}0,063 \\
\left(400^{\circ}\right)\end{array}$ & {$\left[\begin{array}{l}0,03 \\
\left(220^{\prime \prime}\right)\end{array}\right.$} & $\begin{array}{c}0,038 \\
\left(400^{\circ} !\right.\end{array}$ & $\begin{array}{l}0,027^{\circ} \\
\left(320^{\circ}\right)\end{array}$ \\
\hline $\begin{array}{r}\text { Viscosité } \ldots \ldots \\
\text { (centipoises) } \ldots \ldots\end{array}$ & {$\left[\begin{array}{l}0,27 \\
\left(400^{\prime \prime}\right)\end{array}\right.$} & $\begin{array}{l}0,2 \\
\left(400^{\circ}\right)\end{array}$ & $\left(200^{\circ}\right)$ & $\left(\begin{array}{l}2,2 \\
\left(400^{\prime \prime}\right)\end{array}\right)$ & $\begin{array}{l}\ddot{1,5} \\
\left(400^{\circ}\right)\end{array}$ \\
\hline
\end{tabular}


On y remarquera lout de suile que le sodium et l'eutecticue sodium-potassium se délachent de leurs concurrents par leur plus forte chaleur spécifique, leur plus grande conductivité thermique et leur plus faible viscosité, avec une gaumme de tempéralures d'utilisation intéressante à la pression atmosphérique.

Ce sont malheureusement des corps dont chacun connaît la grande activité chimique.

\section{2. - ÉCHANGES DE CHALEUR DANS UN FLUIDE EN CIRCULATION}

\section{1}

Dans un fluide en écoulement turbulent, la chaleur se transmet de deux facons : par conduction moléculaire et par conduction turbulente.

La conduction moléculaire traduit la transmission par chocs du mouvement d'agitation des molécules, agitation qui, en somme, se mesure par la température du corps.

La conduction, ou convection, turbulente, traduit le transport de particules macroscopiques du fluide d'un point à un autre, sous l'effet de la turbulence.

\section{2}

Dans un écoulement turbulent, ce dernier phénomène est prépondérant dès que l'on s'éloigne des parois, masquant presque totalement l'influence de la conduction moléculaire. Près des parois, par contre, la conduction moléculaire pourra intervenir de facon d'autant plus sensible que la turbulence sera plus faible.

\section{3}

Si l'on définit la « diffusivité » d'une grandeur quelconque au sein d'un fuide par le rapport du flux de cette grandeur à son gradient de concentration en un même point, on définira une quantité qui aura pour dimension $\mathrm{L}^{2} \mathrm{~T}^{-1}$, et l'on pourra comparer les diffusivités de deux grandeurs de dimensions différentes telles que quantité de mouvement et quantité de chaleur.

La diffusivité moléculaire de la quantité de mouvement n'est autre que la viscosité cinématique $v$.

Celle de la quantité de chaleur est $k / C$ o où : $k$ est la conductivité thermique,

$C$ la chaleur spécifique.

Le rapport sans dimensions des deux diffusivités est le nombre de Prandtl:

$$
\mathrm{Pr}^{*}=\frac{\mathrm{C} v}{k}(u=p v)
$$

dont la considération permettra de prévoir l'allure des phénomènes de convection pour un ceoulement déterminé.

\section{4}

Un fluide s'écoule dans un tube circulairc.
Contre la paroi, un film laminaire se forme où la diflusion des quantités de mouvement comme de la chaleur n'est que du type moléculaire. La répartition des vitesses y est régie par la viscosité, la répartition des températures par la diffusivité thermique.

Près de l'axe, au contraire, la diffusion turbulente est très largement prépondérante. Vitesses et températures se répartissent selon une même Ioi.

Entre les deux prend place une zone de transition, où les répartitions de vitesses et de températures sont régies à la fois par la diffusivité turbulente et la diffusivité moléculaire.

Si l'on trace sur un diagramme (fig. 1) les courbes de répartition envisagées, on peut les

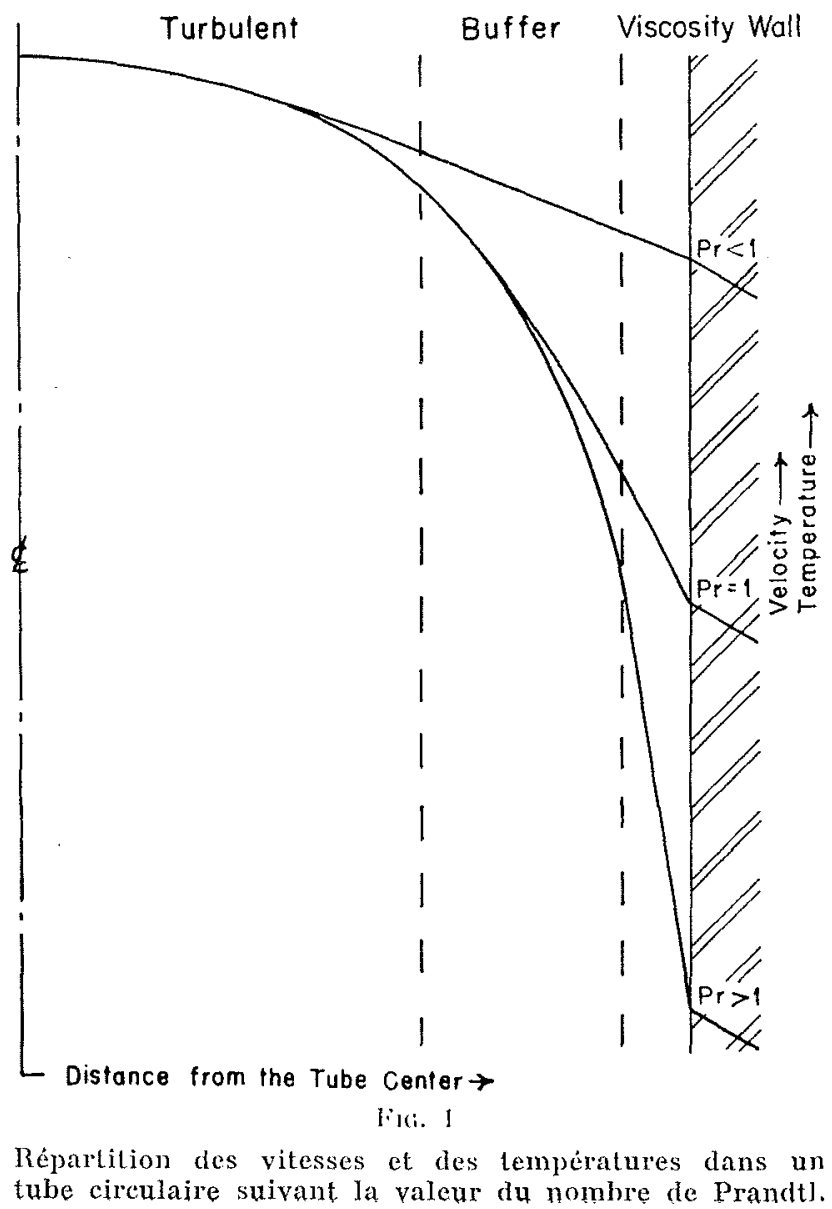


laire coüncider, en choisissanl des échelles convenables, lorsque les diffusivités moléculaires cinématiques et thermiques sont égales, autrement dit lorsque le nombre de Prandtl est égal à 1 . C'est le cas de l'analogie de Reynolds qui reste acceptable lorsque le nombre de Prandtl varie dans certaines limites autour de l'unité. Les gaz. et l'eau à haute température satisfont raisonnablement à cette condition.

Si le nombre de Prandtl est nettement supérieur à $\mathbf{1}$ (fluide mauvais conducteur), les courbes coïncident encore dans le noyau central. Dans le film laminaire, par contre, la courbe de température est en dessous de celle des vitesses, à qui elle se raccorde dans la zone de transition.

Si, enfin, le nombre de Prandtl est nettement infẻrieur à 1 - c'est le cas des métaux liquides - la courbe des températures est très audessus de celle des vitesses dans le film laminaire et dans la zone de transition. Les deux courbes ne coïncident qu'au voisinage de l'axe.

Pour illustrer ces considérations générales, nous avons pris le cas d'un fluide plus chaud que le tube. Dans le cas contraire, on doit considérer la différence $t_{s}-t$ ( $t_{s}$ étant la température de la paroi) au lieu de la température $t$.

\section{5}

Voici les nombres de Prandtl des métaux que nous avons envisagés plus haut:

\begin{tabular}{|c|c|c|c|c|}
\hline $\begin{array}{c}\mathrm{Na} \\
\left(400^{\circ}\right)\end{array}$ & $\begin{array}{c}\mathrm{NaK} \\
\left(400^{\circ}\right)\end{array}$ & $\begin{array}{c}\mathrm{Hg} \\
300^{\circ}\end{array}$ & $\begin{array}{c}\mathrm{Pb} . \\
\left(400^{\circ}\right)\end{array}$ & $\begin{array}{c}\mathrm{Pb} \mathrm{Bi} \\
\left(400^{\circ}\right)\end{array}$ \\
$\ldots \ldots \ldots$ & $\ldots \ldots \ldots$ & $\ldots \ldots \ldots$ & $\ldots \ldots \ldots$ & $\ldots \ldots \ldots$ \\
0,0050 & $0,00(68$ & 0,011 & 0,024 & 0,019 \\
\hline
\end{tabular}

Ils sont tous nettement inférieurs à 1 . On se trouve donc toujours, avec ellx, dans le troisième cas que nous venons de décrire : le transfert de chaleur entre la paroi et l'écoulement central est régi par la conduction.

\section{6}

Les lois empiriques établies pour des fluides tels que l'eau ou les gaz ne s'appliquent plus. On a dû les modifier pour étendre aux métaux liquides leurs champs d'application.. Ces modifications ont été faites par la voie théorique. Des expériences maintenant assez nombreuses ont fourni des résultats encore bien dispersés, conduisant souvent à des coefficients d'échange inférieurs à ceux annoncés par la théorie.

Dans un tube circulaire long, où le flux de chaleur est uniforme le long" de la paroi, la loi théorique dablie par Martinelli comme ralible pour tous les fluides est la suivante:

$$
\frac{k}{h \mathrm{D}}=2 \int_{0}^{1} \frac{\left[\int_{0}^{\mathrm{S}} \mathrm{S}_{1} \mathrm{~V} d \mathrm{~S}_{1}\right]^{2}}{\mathrm{~S}_{2} \mathrm{~K} / K} d \mathrm{~S}_{2}
$$

ou: $k$ est la conductivité thermique;

hl le coefficient d'échange;

$\mathrm{K}$ est la conductivité locale totale (conductivité moléculaire -+ conductivilé de turbulence);

$\mathrm{D}$ Je diamètre;

$S$ la distance à l'axe rapporlée au rayon:

$\mathrm{V}$ est la vitesse Jocale rapportée à la vitesse moyenne.

Si l'on admet que les diffusivités turbulentes des quantités de mouvement et de la chaleur sont égales en tout point, on peut intégrer graphiquement à partir des distributions de vitesses données par Nikuradse et les résultats, comme ceux fournis précédemment par Martinelli, sont traduils correctement par :

$$
\mathrm{Nu}=7+0,025\left((\mathrm{Re} . \mathrm{Pr})^{0,8}\right.
$$

où $\mathrm{Nu}, \mathrm{Re}, \mathrm{Pr}$ sont respectivement les nombres de Nusselt, de Reynolds et de Prandtl.

Dans une telle formule, le coefficient d'échange - ramené à la forme sans dimension - Nu, est la somme de deux termes: l'un, constant, rend compte uniquement de la conduction moléculaire; l'autre, lié au nombre de Prandtl et au nombre de Reynolds, rend compte de la convection turbulente (où, notons-le, intervient également la conduction moléculaire dans l'expression de $\mathrm{Pr}$ ).

Lorsqu'au lieu de transmettre un flux conslant, la paroi du tube est à température constante, on peut établir théoriquement la relation :

$$
\mathrm{Nu}=5+0,025(\operatorname{Re} \operatorname{Pr})^{0,8}
$$

ne différant de (2) que par la valeur de la constante relative à la conduction.

Il existe d'autres relations théoriques établies pour le cas d'espaces annulaires, de plaques parallèles, etc.

En réalité, tous les résultats d'essais publiés indiquent des coefficients d'échange sensillscment inférieurs aux valeurs théoriques. Un grand nombre de résultats, ramenés à des conditions de mesure identiques, ont été rassemblés par Lubarsky et KaUfman (*), qui en ont déduit une loi entièrement empirique sous la forme :

$$
\mathrm{Nu}=0,625 \mathrm{Pe}^{0.4}
$$

Par rapport à la formule théorique (2), on constate un écart par défaut de $50 \%$ pour des nombres de Péclet de lordre de 100, de $30 \%$ pour des nombres de Péclet de l'ordre de 1.000 . 


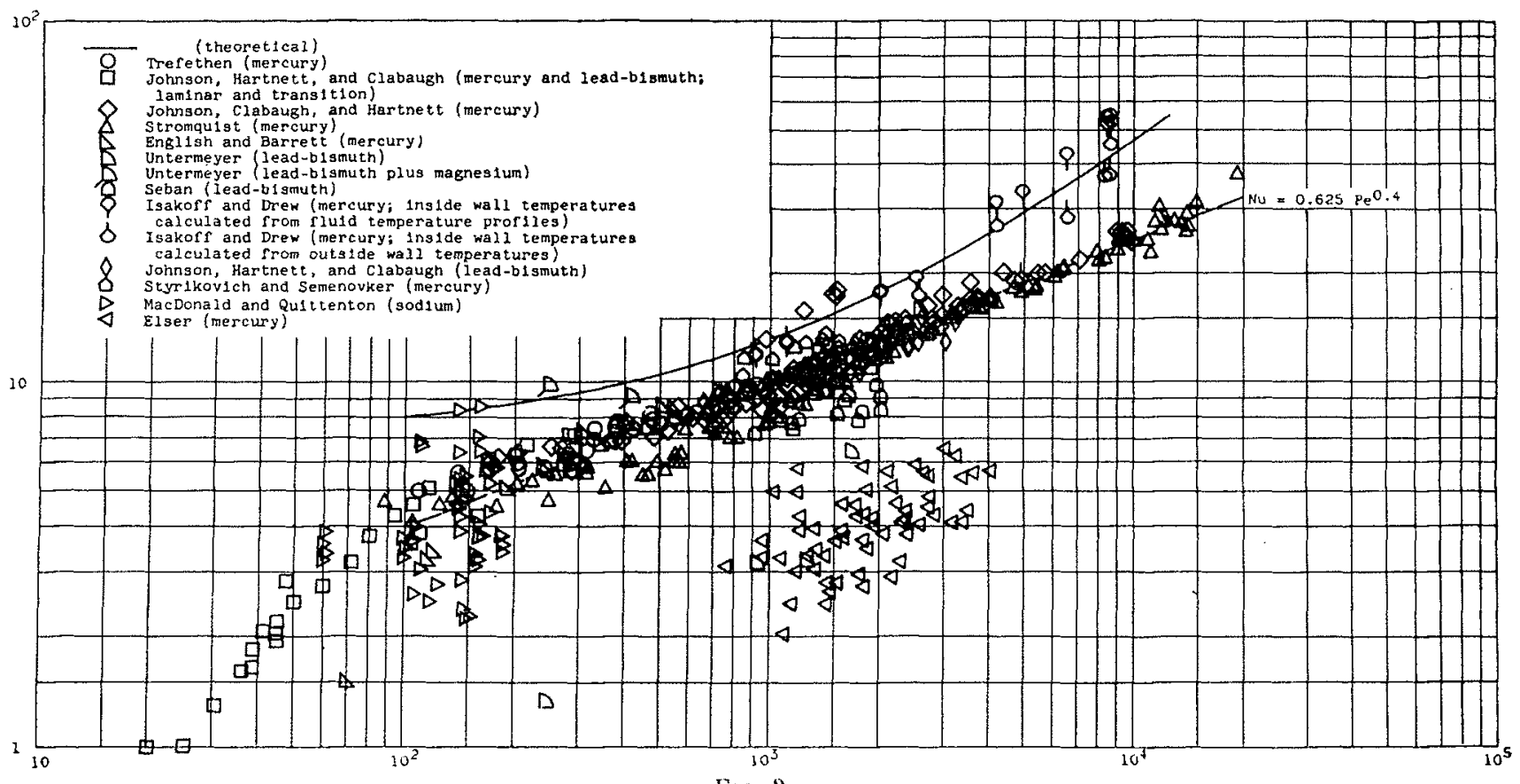

Fig. 2

Résultats expérimentaux rassemblés par Lubarsky et Kaufman.

$\mathrm{Nu}$.

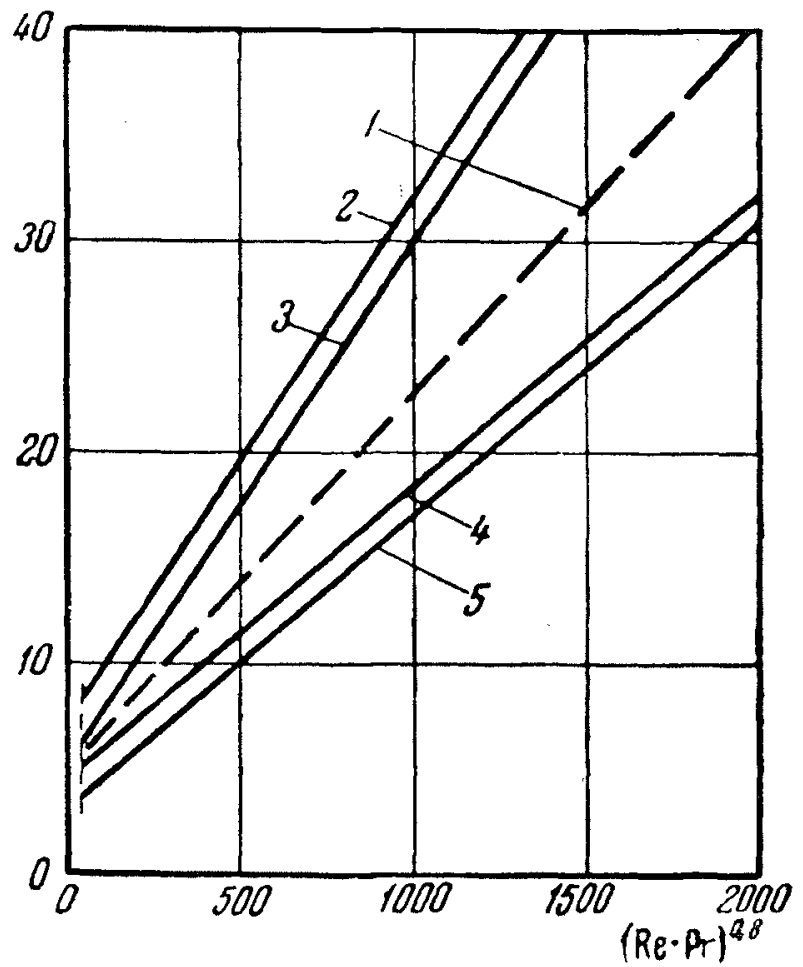

Fig. 3

Comparaison des résultats :

1. Divger (1954). Théorie de Lyon avec correction expérimentale.

2. Théorie de Lyon (1951).

3. Théorie de Seban (1951).

4. Expérience de l'Ac. des Sc. de l'U.R.S.S., surfaces propres.

5. Expérience de l'Ac. des Sc. de l'U.R.S.S., surfaces non désoxydées.
A la conférence de Genève, des chercheurs soviétiques $\left(^{\star}\right)$ ont rendu compte d'expériences couvrant un domaine assez vaste. Là encore, les résultats se montrent tous très au-dessous des résullats théoriques. Ils peuvent se traduire par l'une ou l'autre des formules :

$$
\begin{aligned}
& \mathrm{Nu}=4,5+0,014\left(\operatorname{Re} \times \mathrm{Pr}^{0,8}\right. \\
& \mathrm{Nu}=3+0,014\left(\operatorname{Re} \times \mathrm{Pr}^{0,8}\right)^{0,8}
\end{aligned}
$$

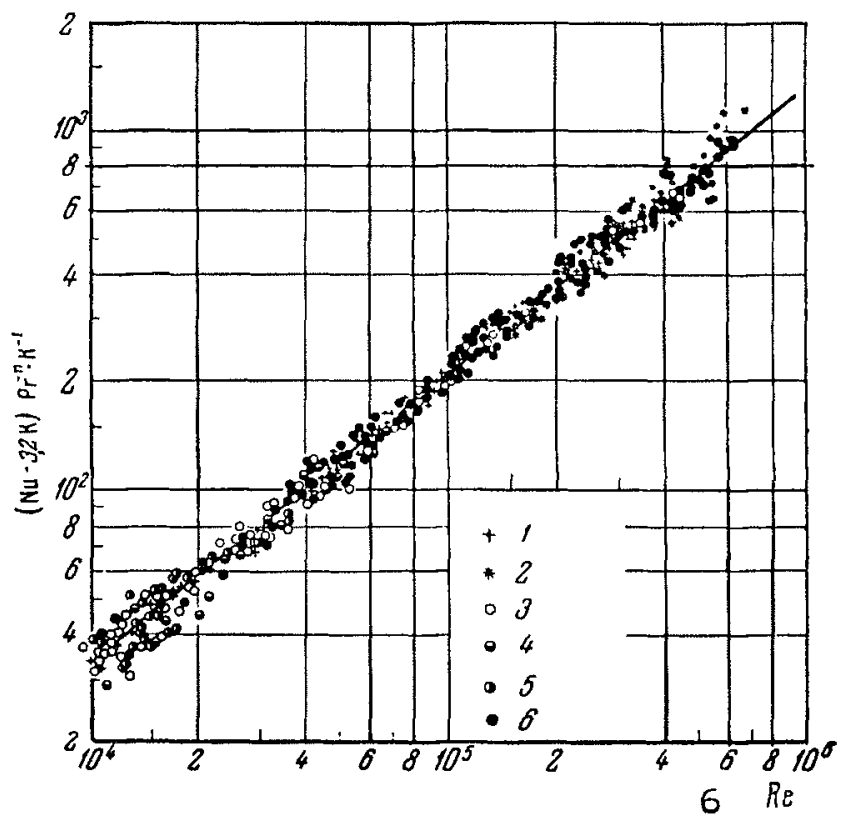

Fig. 4.

Résultats regroupés pour différents fluides :

1. Air. - 2. Vapeur surchauffée. - 3. Eau. - 4. Kérosène acétone. - 5. Huiles minérales. - 6. Métaux liquides. 
selon que la paroi est ou non soigneusement désoxydée avant l'expérience.

La figure 3 montre comment se placent les différents résultats, théoriques et expérimentaux.

La figure 4 regroupe sur une courbe unique un grand nombre de points expérimentaux relatifs à des fluides très divers, selon une formule « universelle» de la forme :

$$
\mathrm{Nu}=\alpha+\beta \operatorname{Re}^{0,8} \cdot \mathrm{Pr}^{n}
$$

où $n$ est une fonction uniforme du nombre de Prandtl, qu'en pratique on peut prendre égal à 0,43 pour les fluides usuels à faible conductivité (gaz, eau, huiles...) et à 0,90 pour les métaux fondus.

\section{7}

Plusieurs raisons sont envisagées pour expliquer le désaccord si fréquent, et souvent si important, entre théorie et expérience dans le cas des métaux liquides. Aucune n'est jugée convaincante, ou du moins suffisante, par l'ensemble des expérimentateurs. Voici celles qu'on a proposées :

1) le liquide ne mouille pas la paroi;

2) la paroi se couvre d'un dépôt;

3) le liquide entraîne du gaz;

4) on devrait tenir compte de la conduction longitudinale dans le liquide.

Si les expérimentateurs soviétiques attribuent entièrement aux deux premières raisons le désaccord constaté, l'opinion de LyoN est plus nuancée. Il considère, d'abord, qu'aux faibles vitesses l'hypothèse du flux de chaleur constant peut être mise en défaut par l'existence d'une certaine convection naturelle, ou encore par la libération et l'accumulation, sur des zones mal mouillées de la paroi, de gaz dissous ou entraînés. Cela pourrait, selon lui, rendre compte de la diminution importante du coefficient d'échange pour des nombres de Péclet inférieurs à 100 .

D'autre part, il convient que la plus grande faiblesse de l'intégration qui aboutit à la formule théorique réside dans le postulat égalant les diffusivités turbulentes des quantités de mouvement et de la chaleur.

Si plusieurs auteurs ont tenté d'approcher mieux la réalité en remplaçant la diffusivité des quantités de mouvement par celle de la « vorticité », ou en modifiant la théorie de la longueur de mélange, ou encore en attribuant aux forts gradients de vitesse une influence sur la conductivité thermique, nous suivrons plutôt l'opinion de Lyon lorsqu'il estime que les choses s'expliqueront mieux lorsqu'on disposera d'un ensemble expérimental plus poussé et plus cohérent.

\section{8}

Il faut signaler dans cet ordre d'idée les mesures effectuées par IsAkoff et Drew : ce sont, du moins d'après ce qui fut publié jusqu'à présent, les seuls expérimentateurs qui aient relevé, dans un échangeur à métal liquide, les profils de vitesse et de température. Il s'agit là d'un genre d'expérimentation extrêmement délicat, mais qui seul permettra sans doute, avant de faire appel à une connaissance approfondie de la structure fine de la turbulence, d'élaborer une théorie peut-être encore schématique mais cejendant mieux représentative des phènomènes réels. La figure 5 montre les profils de température obte-

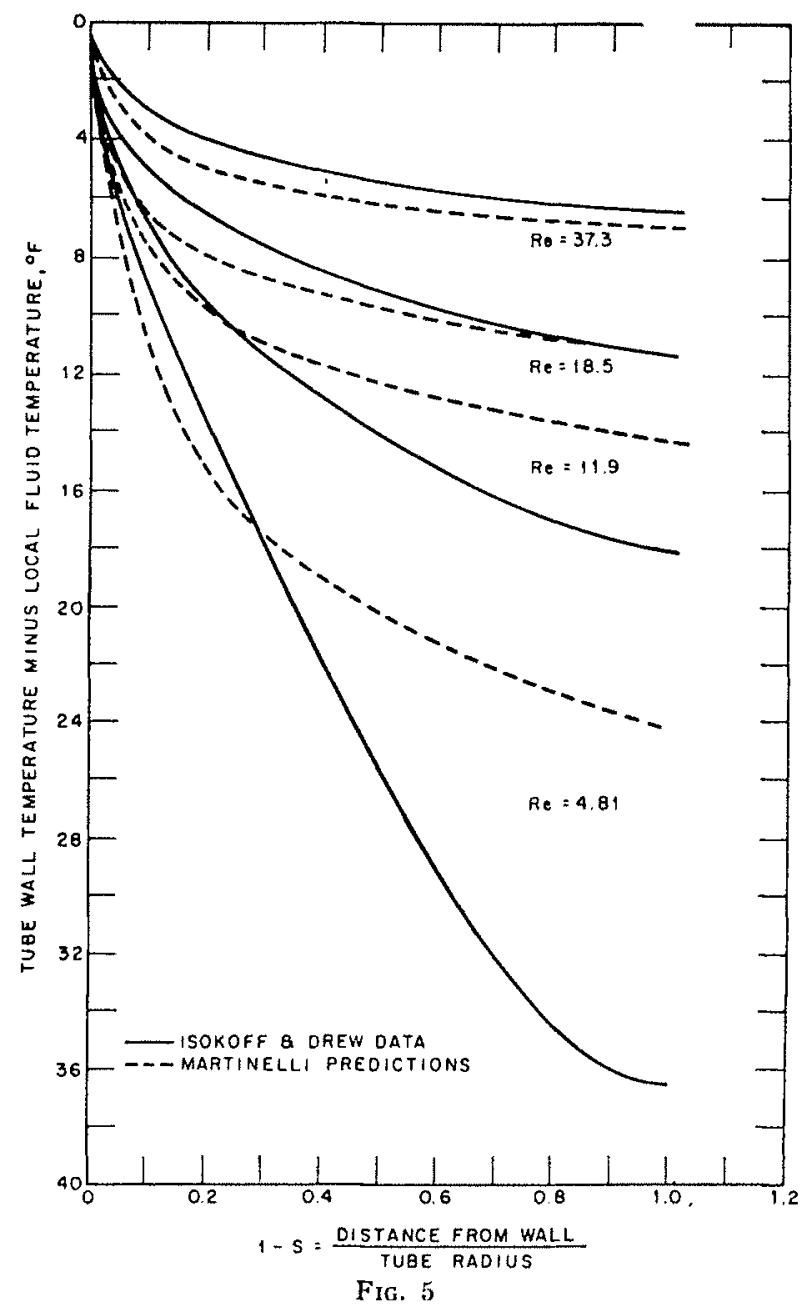

Comparaison des distributions de temperatures expérimentales (mercure, $\mathrm{Pr}=0,0239$ ) avec celles prévues par Martinelli, d'après Isakoff et Drew.

nus par ISAKOFF et DREw, comparés aux profils théoriques prévus par Mantinela pour du mercure (Pr. 0,0239). Bien que les coefficients d'échange globaux obtenus au cours de ces essais soient assez proches de ceux ealculés par la formule (2), on constate un très net désaccord entre les deux séries de profils de température. 


\section{9}

Enfin, un facteur important peut influencer fortement les résultats globaux de mesures: la géométrie exacte des conduils où sont mesurés les coefficients d'échange. Un défaut, même léger, de centrage ou de symétrie a pour conséquence la non-uniformité des températures de paroi dans une section droite de l'écoulement. L'influence de ces écarts de température sur les coefficients d'échange globaux est d'autant plus grande, relativement, que le nombre de Prandtl est plus faible et, dans le cas des métaux liquides, ce pourrait être une explication à la dispersion des résultats.

\subsection{0}

Les études théoriques et expérimentales ne se sont pas limitées à la circulation dans des tuyaux circulaires. Les écoulements entre plaques planes parallèles ont notamment fait l'objet d'ćtu- des varices, ainsi que ceux dans des espaces annulaires. D'une façon générale, les conclusions restent les mêmes : les théories actuelles ne rendent compte que d'une facon assez approchée, et toujours par excès, des résultats expérimentaux. Par contre, ces derniers sont maintenant assez nombreux pour permettre, par des formules empiriques, de calculer un circuit de refroidissement avec une approximation raisonnable,

\subsection{1}

Il faut enfin mentionuer un problime essentiellement différent, celui de la convection naturelle. Nous ne l'exposons pas ici. Des études théoriques assez complètes en ont été faites dans les deux cas de l'écoulement laminaire et de l'écoulement turbulent. Mais les données expérimentales manquent presque totalement dans la littérature pour en vérifier la validité.

\section{3. - SECURITE - CORROSION - POMPAGE}

\section{1}

Nous ne faisons que signaler ces problèmes, qu'il est important d'avoir à l'esprit pour s'expliquer les difficultés que l'on doit surmonter lorsqu'on entreprend toute étude expérimentale des écoulements et des échanges de chaleur en métaux liquides.

\section{2}

Les utilisateurs éventuels de métaux liquides ont résolument fixé leur choix sur le sodium et sur les alliages sodium-potassium; ceux-ci présentent sur le sodium pur l'avantage d'être liquides à température plus basse :

$19^{\circ} \mathrm{C}$ pour $\mathrm{NaK}$ à $44 \%$ de $\mathrm{K}$ (en poids), $-11{ }^{\circ} \mathrm{C}$ pour l'eutectique à $78 \%$ de $\mathrm{K}$ (en poids),

$97,8{ }^{\circ} \mathrm{C}$ pour le sodium pur.

Ce choix résulte de la conductivité et de la chaleur spécifique relativement élevées de ces corps.

Mais ils se caractérisent par une grande affinité pour l'oxygène qui oblige à des précautions considérables concernant l'étanchẻité des circuits. Plusieurs raisons à cela. D'abord le risque d'inflammation spontanée à l'air, ou de réaction violente au contact de l'eau, dans un échangeur par exemple. Ensuite la formation d'oxyde en présence de trace d'oxygène ou d'un corps oxygéné moins réducteur. Cet oxyde ayant une température de fusion beaucoup plus élevee, peut former un film thermiquement isolant réduisant considérablement les coefficients d'échange ou, ce qui est plus certain, précipiter dans les zones les plus froides et obstruer complètement les circuits.

Il est donc nécessairc, non seulement de réaliser des circuits parfaitement étanches, mais aussi de choisir avec un soin tout particulier les matériaux entrant dans leur constitution : certains risques de corrosion ou de rupture qui pour l'cau peuvent être acceptés ne peuvent plus l'être dans ce cas.

\section{3}

Les techniciens américains ont, semble-t-il, ćtudié très extensivement la tenue au sodium de nombreux métaux et alliages à des températures diverses. Pratiquement, les nombreux alliages de fer, nickel, chrome, depuis le 18/8 courant jusqu'à l'inconel, résistent bien jusqu'à au moins $900^{\circ} \mathrm{C}$. De même, les alliages à haute teneur en cobalt, et des métaux réfractaires comme le chrome, le tungstène, le molybdène. D'une façon générale, les aciers au carbone, au phosphore, les fontes, les alliages de cuivre, sont à proscrire au-dessus de $500^{\circ} \mathrm{C}$.

\section{4}

La nécessité d'une parfaite étanchèité, de classe «nucléaire » pourrait-on dire, donne au problème du pompage une importante toute spéciale. M. Hermant parlera demain des deux catégories de pompes utilisables, mécaniques et électromagnétiques. 


\section{4. - CONCLUSION}

C'est bien la recherche d'une solution élégante aux difficiles problèmes d'échange de chaleur que pose, dans certains cas, l'utilisation de l'énergie atomique, qui a suscité dans tous les pays un intérêt considérable pour les métaux liquides, notamment le sodium et les alliages
NaK, Mais il n'est pas plus douteux qu'une meilleure connaissance de leur's propriétés - et surtout une plus grande expérience de leur manipulation - permettra d'en développer des applications intéressantes dans d'autres domaines.

BIBLIOGRAPHIE

Liquid Metals Handbook. - Atomic Energy Commission. Depart. of the Navy. Juin 1952.

Liquid Metals Handbook. - Sodium-NaK Supplement. Depart. of the Navy. Juillet 1955.

Martineldi R. C. - Heat Trausfer to Molten Metals. Trans. A.S.M.E., 9 (pp. 947-959).
LYon R. N. - Conférence de Genc̀ve. Août 1955, p. 120. M. A. Mrnheyev et al. - Conférence de Genève. Aoùt 1955, p. 639 .

IsAKOFF et DREw. - Heat Transter in Turbulent flow in Mercury. General Discussion on Heat Transfer. Inst. Mech. Eng. et A.S.M.E., 405-409 (1951).

\section{COMMENTAIRE DE M. GIBRAT}

Président

M. le Président remercie M. Braudeau de celle revue des propriétés thermodynamiques des métanx liquides, faite d'une façon complète pour la première fois.

M. le Président termine par une anecdote personnelle : au cours d'une visite récente à l'un des principaux laboratoires américains, il a vu une installation expérimentale à grande échelle constituée par une « imitation : de réacteur, sans élément radio-actif, baignant dans du sodium liquide en circulation et munie d'appareils de mesures enregistreurs; malheureusement, ainsi que le montrail une bride de tuyauterie non assemblée, Ie système était arrêté pour cause d'incident.

« Il y a donc encore des progrès à faire dans cette voie, poursuit M. Gimnat, de mème que sur le plan théorique, l'intervention de M. Fortier dans la discussion de la communication de $M$. Jouty fait pressentir que les principes ne sont pas inçbranlables. On doit cependant féliciter tous les conférenciers qui ont su traiter suus des aspects très divers et brillamment la question mise à l'ordre du jour de ces séances. Je remercie aussi vivement les participants. 》

La séance est levée à 18 h 10 . 